\section{Intraoperative pulmonary tumour embolism during hepatectomy}

To the Editor:

We were interested in the case of hepatic tumour embolism reported by Blanloeil et al, ${ }^{1}$ and their comment that it had not been previously reported to have occurred intraoperatively.

We would like to report a case of a nine-monthold infant who presented with a large hepatoblastoma. The patient was anaesthetized and cooled to $32^{\circ} \mathrm{C}$, which is our usual practice for such operations. The operation proceeded satisfactorily with blood loss being replaced. The right hepatic artery, portal vein and hepatic duct were divided. During the dissection of the hepatic vein about $100 \mathrm{mls}$ of blood was lost which was rapidly replaced. Shortly afterwards the blood pressure declined, hypoxia developed $\left(\mathrm{PaO}_{2} 30 \mathrm{mmHg}\right)$ and cardiovascular collapse occursed. Despite further blood transfusion and resuscitation for over an hour the patient failed to respond and died. At postmortem multiple, large tumour emboli were found throughout the pulmonary vascular tree.

During hepatic resections hypothermia provides some cerebral protection should cardiac output decline. This may result from blood loss or decreased venous return if inferior vena caval occlusion becomes necessary to repair vena caval perforation. Placing slings around the inferior vena cava is a helpful safeguard in the event of such difficulty. Although it might be useful if embolisation is considered likely it would be hard to detect emboli until it was too late as it was in our case.

If tumour has already invaded the vena cava then operation using cardiopulmonary bypass would be necessary. There was no evidence from preoperative radiological investigations or ultrasound that the inferior vena cava was involved in our patient nor was there evidence at postmortem of tumour spread into the vena cava. We have successfully operated with bypass on another child with Wilms tumour invading the vena cava. In that case the intravascular tumour was removed as a solid plug.
Fortunately massive tumour embolism during hepatic resection is rare, this being the only one we have had in a series of 46 patients.

\author{
Barbara J. Main \\ T.C.K. Brown \\ P.G. Jones \\ Departments of Anaesthesia and Surgery \\ Royal Children's Hospital \\ Melboume, Australia
}

\section{REFERENCE}

1 Blanloeil Y, Paineau J, Visselt J, Dixneuf B. Intraoperative pulmonary tumour embolism after hepatectomy for liver carcinoma. Can Anaesth Soc J 1983; 30: 69-71.

\section{Naloxone and its antagonism of anaesthesia and analgesia}

To the Editor:

The paper by Freye et al. is of interest, ${ }^{1}$ but I feel certain statements and assumptions made by these authors should be challenged.

They state that the opiate antagonist naloxone (Nx) can antagonise certain non-opiate-induced effects, but do not qualify this statement by adding that these effects may be governed by $\mathrm{Nx}$-sensitive endogenous opioid activity. They then go on to cite studies where non-opiate substances such as diazepam, alcohol and nitrous oxide have been shown to exhibit Nx-sensitive actions.

They infer from this that $\mathrm{Nx}$ sensitivity may not necessarily give an indication of opiate involvement. Although $\mathrm{Nx}$ sensitivity per se does not give unequivocal proof of opiate or opioid activity, ${ }^{2}$ it is most suggestive particularly at low doses of $\mathrm{Nx}$. The examples that these authors cite are particularly misleading. First they do not make a clear distinction between anaesthesia and analgesia, which is most important ${ }^{3}$ since different mechanisms may mediate these two states. ${ }^{4}$ 